\title{
A single-cell transcriptomic atlas of the developing chicken limb
}

\author{
Christian Feregrino ${ }^{1}$, Fabio Sacher ${ }^{1}$, Oren Parnas ${ }^{2,3}$ and Patrick Tschopp ${ }^{1 *}$ (D)
}

\begin{abstract}
Background: Through precise implementation of distinct cell type specification programs, differentially regulated in both space and time, complex patterns emerge during organogenesis. Thanks to its easy experimental accessibility, the developing chicken limb has long served as a paradigm to study vertebrate pattern formation. Through decades' worth of research, we now have a firm grasp on the molecular mechanisms driving limb formation at the tissue-level. However, to elucidate the dynamic interplay between transcriptional cell type specification programs and pattern formation at its relevant cellular scale, we lack appropriately resolved molecular data at the genomewide level. Here, making use of droplet-based single-cell RNA-sequencing, we catalogue the developmental emergence of distinct tissue types and their transcriptome dynamics in the distal chicken limb, the so-called autopod, at cellular resolution.

Results: Using single-cell RNA-sequencing technology, we sequenced a total of 17,628 cells coming from three key developmental stages of chicken autopod patterning. Overall, we identified 23 cell populations with distinct transcriptional profiles. Amongst them were small, albeit essential populations like the apical ectodermal ridge, demonstrating the ability to detect even rare cell types. Moreover, we uncovered the existence of molecularly distinct sub-populations within previously defined compartments of the developing limb, some of which have important signaling functions during autopod pattern formation. Finally, we inferred gene co-expression modules that coincide with distinct tissue types across developmental time, and used them to track patterning-relevant cell populations of the forming digits.

Conclusions: We provide a comprehensive functional genomics resource to study the molecular effectors of chicken limb patterning at cellular resolution. Our single-cell transcriptomic atlas captures all major cell populations of the developing autopod, and highlights the transcriptional complexity in many of its components. Finally, integrating our data-set with other single-cell transcriptomics resources will enable researchers to assess molecular similarities in orthologous cell types across the major tetrapod clades, and provide an extensive candidate gene list to functionally test cell-type-specific drivers of limb morphological diversification.
\end{abstract}

Keywords: scRNA-seq, Gene expression, Cellular transcriptomics, Autopod patterning, Digits, Interdigit, Perichondrium, Phalanges

\section{Background}

Embryonic pattern formation relies on the tight coordination of numerous developmental processes, across multiple scales of complexity. From seemingly homogenous progenitor populations, different cell types get specified and arranged in intricate patterns, to give rise to functional tissues and organs. As progenitors mostly share a common genome, this phenotypic specialization relies

\footnotetext{
* Correspondence: patrick.tschopp@unibas.ch

'DUW Zoology, University of Basel, Vesalgasse 1, CH-4051 Basel, Switzerland Full list of author information is available at the end of the article
}

on the precise execution of distinct gene regulatory networks, to enable cell type specification and ensuing pattern formation [1-3]. Slight deviations in these processes contribute to morphological variations within natural populations. More profound aberrations, however, can cause malformations and ultimately result in death of the embryo. To buffer such fragile balance, many cell type specification and pattering processes rely on complex feedback mechanisms, through tightly interconnected molecular loops between spatially distinct signaling centers [4-6]. Hence, integration of multiple

(c) The Author(s). 2019 Open Access This article is distributed under the terms of the Creative Commons Attribution 4.0 International License (http://creativecommons.org/licenses/by/4.0/), which permits unrestricted use, distribution, and 
signaling pathways across space and time defines a molecular coordinate grid to instruct organogenesis at the tissue level. Ultimately, however, these multifaceted signaling inputs have to be incorporated at the cellular level, via cell type-specifying gene regulatory networks, as progenitor cells undergo spatially and temporally defined cell fate decisions to contribute to proper pattern formation.

Tetrapod limb development has long served as a model to study the genetic and molecular underpinnings of vertebrate pattern formation. Due to its non-essentiality for embryo survival, many fetuses carrying mutations that affect limb development make it to full term. Accordingly, human geneticists have been able to accumulate an impressive catalogue of candidate genes for limb patterning [7-9]. Combined with the easy accessibility of the limb in chicken embryos, and molecular genetic tools in the mouse, decades of experimental work have resulted in an in-depth understanding of many of the molecular mechanisms driving limb formation at the tissue scale [5]. Moreover, given the profound morphological diversifications the basic limb structure has experienced in numerous tetrapod clades, limb development has long attracted the interests of comparative developmental biologists using 'EvoDevo' approaches [10]. This holds especially true for the most distal portion of the limb, the autopod, i.e. hands and feet. There, species-specific adaptations to distinct modes of locomotion have resulted in a diverse array of digit number formulas and individualized digit patterns [11-14].

Early in development, proliferation of a lateral plate mesoderm (LPM)-derived mesenchymal progenitor population drives overall limb bud outgrowth. Signaling crosstalk with a specialized structure of the distal overlaying ectoderm, the apical ectodermal ridge (AER), controls these dynamics. Concurrently, the major embryonic axes of the limb are defined by the coordinated action of multiple signaling centers [reviewed in 5]. As development progresses, LPM-derived progenitors start to differentiate into skeletal and other connective tissue types [15-17], while muscles cells originating from the somites migrate into the limb bud to complement formation of the musculoskeletal apparatus [18, 19]. For autopod pattern formation, digit numbers and identities are first defined by posteriorly restricted sonic hedgehog $(\mathrm{SHH})$ activity, and altered by modulations therein $([10,14,20]$, reviwed in [21]). Digit elongation then relies on a specialized distal progenitor population, which supports outgrowth of individual digit bones, the phalanges [22, 23]. Digit-specific phalanx-formulas, and their stereotypic connection patterns via synovial joints, are established by signals emanating from the posterior interdigit mesenchyme [24, 25].

In this study, capitalizing on the power of droplet-based single-cell RNA-sequencing, we resolve the underlying transcriptional dynamics of autopod tissue formation and pattern emergence at single-cell resolution, across three stages of chicken hindlimb development. In total, we present transcriptomic data for 17,628 cells, allowing us to identify all major tissue types of the developing limb, as well as a substantial amount of molecular heterogeneity therein. Through weighted correlation network analysis, we define distinct gene co-expression modules that track corresponding tissue types across developmental time. Finally, we focus on the molecular make-up of cell populations involved in digit pattern formation and, hence, putative drivers of morphological diversification in the autopod.

Collectively, we present a comprehensive genomics resource that for the first time reveals the transcriptome dynamics of the developing chicken foot at the cellular level. Our study identifies a range of marker genes in co-expression modules of patterning-relevant cell populations. Thereby, we provide an extensive catalogue of candidate genes for functional follow-up studies to elucidate the molecular mechanisms of autopod pattern formation and diversification.

\section{Results}

Singe-cell sampling of the developing distal chicken limb

To follow the appearance of patterning-relevant cell populations and their associated transcriptome dynamics, we sampled three developmental stages of the embryonic chicken foot: stage Hamburger-Hamilton 25 (HH25, 4.5 days of development), stage HH29 ( 6 days of development) and stage HH31 ( 7 days of development). This time window spans key morphogenetic events that drive species-specific patterns in the developing autopod, particularly for the skeletal apparatus and its associated tissues. Namely, stage $\mathrm{HH} 25$ is dominated by overall autopod outgrowth and delineation of the main embryonic axes, at HH29 digit-specific patterns differentiate, and at HH31 digit elongation is phasing out. We designed our tissue sampling strategies accordingly. At $\mathrm{HH} 25$, we captured the entire distal part of the growing limb (Fig. 1a), at $\mathrm{HH} 29$ we dissected two digits with distinct skeletal formulas, digit 3 and 4 , as well as their adjacent interdigit mesenchyme (Fig. 1b), and at HH31 we focused on the tip of digit 4 with its growth-relevant progenitor population (Fig. 1c). We dissociated the micro-dissected tissue pieces using enzymatic digest combined with mechanical shearing and prepared single-cell suspensions for droplet-based high-throughput single-cell RNA-sequencing (10X Genomics and Drop-Seq [26, 27]). Using the corresponding bioinformatics pipelines, the resulting Next-Generation Sequencing libraries were mapped to the chicken genome, de-multiplexed according to their cellular barcodes and quantified to generate gene/cell UMI (unique molecular identifier) count tables. In total, we sampled over 17,000 cells and obtained single-cell transcriptomic profiles for 5982 (HH25), 6823 (HH29) and 4823 (HH31) individual 


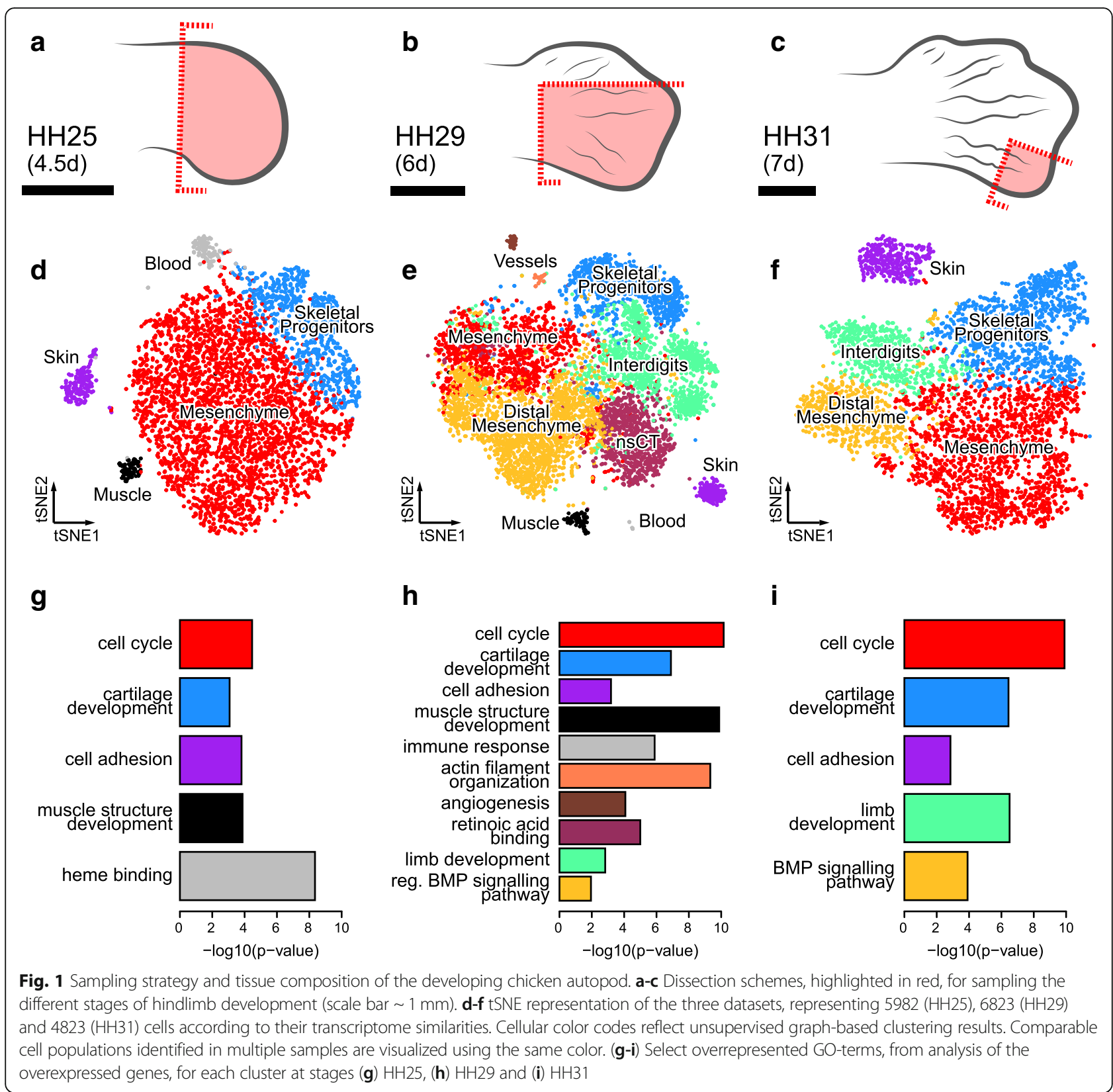

cells, respectively (Additional file 1: Figure S1a). Qualitybased exclusion of single-cell transcriptomes was implemented based on mean library size, percentage of mitochondrial reads and number of genes detected per cell. Additionally, data normalization as well as batch and cell cycle corrections were performed (for details, please refer to the Methods section). On average, we detected 2879 UMIs and 1081 genes per cell (Additional file 1: Figure S1b,c).

\section{Autopod tissue composition at cellular resolution}

Using unsupervised graph-based clustering, we identified 5, 10 and 5 clusters at stages $\mathrm{HH} 25, \mathrm{HH} 29$ and HH31, respectively. Projecting these clusters onto stage-specific
tSNE (t-Distributed Stochastic Neighbor Embedding [28]), plots of our cellular transcriptomes revealed the presence of a dominant bulk of cells, with varying degrees of sub-structure, as well as distinct outlier groups (Fig. $1 \mathrm{~d}-\mathrm{f}$ ). Based on the expression of known marker genes and gene ontology (GO)-term enrichment analyses, we were able to attribute these broadly defined cell populations to distinct tissue types (Fig. 1g-i, Additional file 1: Figure S1a and Figure S2a-c). At stage HH25, they comprise a largely undifferentiated and proliferating mesenchymal population (red), early skeletal progenitors (blue), muscle cells invading the limb (black), as well as skin (purple) and blood cells (grey) (Fig. 1d,g). We recovered cell 
populations corresponding to those same five tissue types in our HH29 sample, with the exception that the "blood cluster" was now dominated by white blood cells and not erythrocytes. Additionally, we identified cell populations matching the interdigit mesenchyme (green), non-skeletal connective tissue (nsCT, maroon), cells enriched for markers of the very distal margin of the autopod mesoderm ("distal mesenchyme", yellow), as well as endothelial (brown) and smooth muscle (orange) cells of the forming blood vessels (Fig. 1e,h). At stage HH31, we again find a largely undifferentiated mesenchymal population, the interdigit and distal margin mesenchyme, skeletal and skin cells (Fig. 1f,i). As expected according to our sampling strategy, for spatial and/or temporal context, we did not find all cell populations in every dataset. For example, while sample $\mathrm{HH} 25$ is biggest in relative size to the autopod, it is the earliest stage and thus predictably displayed the lowest cellular complexity. Conversely, even though development and cell type specification have advanced furthest in our HH31 sample, microdissection of only the tip of digit 4 prevented the capture of more diverse cell populations (Fig. 1c). Hence, our most complex dataset, in terms of cell number and tissue types identified, is from stage HH29. Collectively, using broad graph-based clustering and molecular profiling on our single-cell transcriptomics data, we catalogued the tissue composition of the developing autopod with cellular resolution, across three developmental stages.

\section{Fine-scale clustering and marker gene expression across developmental time}

Although all expected major tissue types were recovered in our primary analyses, smaller cell populations, some well known to be essential for limb outgrowth and patterning, remained elusive. Hence, given our sampling depth, we next examined our data for additional sub-structure. Indeed, upon closer inspection using finer-tuned clustering parameters, we did find additional sub-populations with distinct transcriptional signatures (Fig. 2a-c, Additional file 1: Figure S1a). Based on differential expression analyses, we identified marker genes for each of these sub-populations (Additional file 2, Additional file 3, Additional file 4). Certain sub-population/marker gene-combinations appeared to be conserved in all three samples, thereby allowing us to assign cellular equivalencies across developmental time (Fig. 2d-f). A subset of marker genes only showed loosely restricted expression patterns, likely a reflection of the largely undifferentiated state of the corresponding sub-population. For example, PRRX1, a well-established marker of the limb mesenchyme $[16,29,30]$, and $P C N A$, active during DNA replication in proliferating cells [31], showed varying levels of expression beyond the proliferating mesenchyme sub-clusters. Such transcriptional ambiguities, however, seemed progressively lost, as mesenchymal progenitors committed to the different skeletal and non-skeletal lineages that define the emerging autopod patterns (Fig. 2d-f). As expected, cell sub-populations residing outside the LPM-lineage showed more pronounced transcriptome individualizations. For example, at $\mathrm{HH} 25$ the ectodermal 'skin' population got split into two distinct subclusters, one representing the bulk amount of the embryonic skin covering the autopod (sub-cluster 8), and the other corresponding to the apical ectodermal ridge (sub-cluster 7). Expression of its canonical marker FGF8 and other highly enriched genes clearly established AER identity, demonstrating that even small cell populations can be successfully captured (Fig. 2d).

Gene co-expression modules and corresponding tissue types To gain further insights into the regulatory programs that maintain these transcriptional signatures, and explore their potential biological significance, we tested for the occurrence of transcriptome-wide gene co-expression patterns using weighted correlation network analysis (WGCNA) [32]. This approach consists of an unsupervised clustering of genes based on their expression pattern across all cells, irrespective of the assigned cell or tissue type. In order to comprehensively screen for relevant gene co-expression modules, we conducted the analysis in our transcriptionally most complex sample at stage HH29. Starting with genes that showed high levels and variation of expression, we calculated an adjacency matrix and its topological overlap to construct a hierarchical tree. The resulting tree was cut to obtain a first set of gene co-expression modules. We then computed the first principal component of each module, to define so-called 'module eigengenes'. For each individual gene, correlation to the respective eigengenes was used to assess module membership. Genes not significantly correlated with any eigengene were discarded, after which the entire process was repeated iteratively with a reduced gene set. Eventually, we identified a total of 836 genes grouped in 16 distinct gene co-expression modules, each designated by a color (Fig. 3a). Final module sizes ranged from 15 to 215 genes (Additional file 5).

On a cell-by-cell basis, we calculated the average expression for each of the co-expression modules and visualized their distribution on our stage HH29 tSNE plot (Additional file 1: Figure S3). Compared to our initial clustering of sample $\mathrm{HH} 29$, we found co-expression modules specifically enriched in the following cell populations: blood cells (module Black), skin (Blue), blood vessel endothelium (Brown), nsCT (Darkgrey), distal mesenchyme (Magenta), chondrocytes (Red and Turquoise) and muscle (Yellow). Interestingly, GO-terms associated with more broadly distributed modules enabled us to attribute the sub-clustering structure of certain tissues to particular biological processes. For example, HH29 mesenchyme sub-cluster 5 showed higher activity 


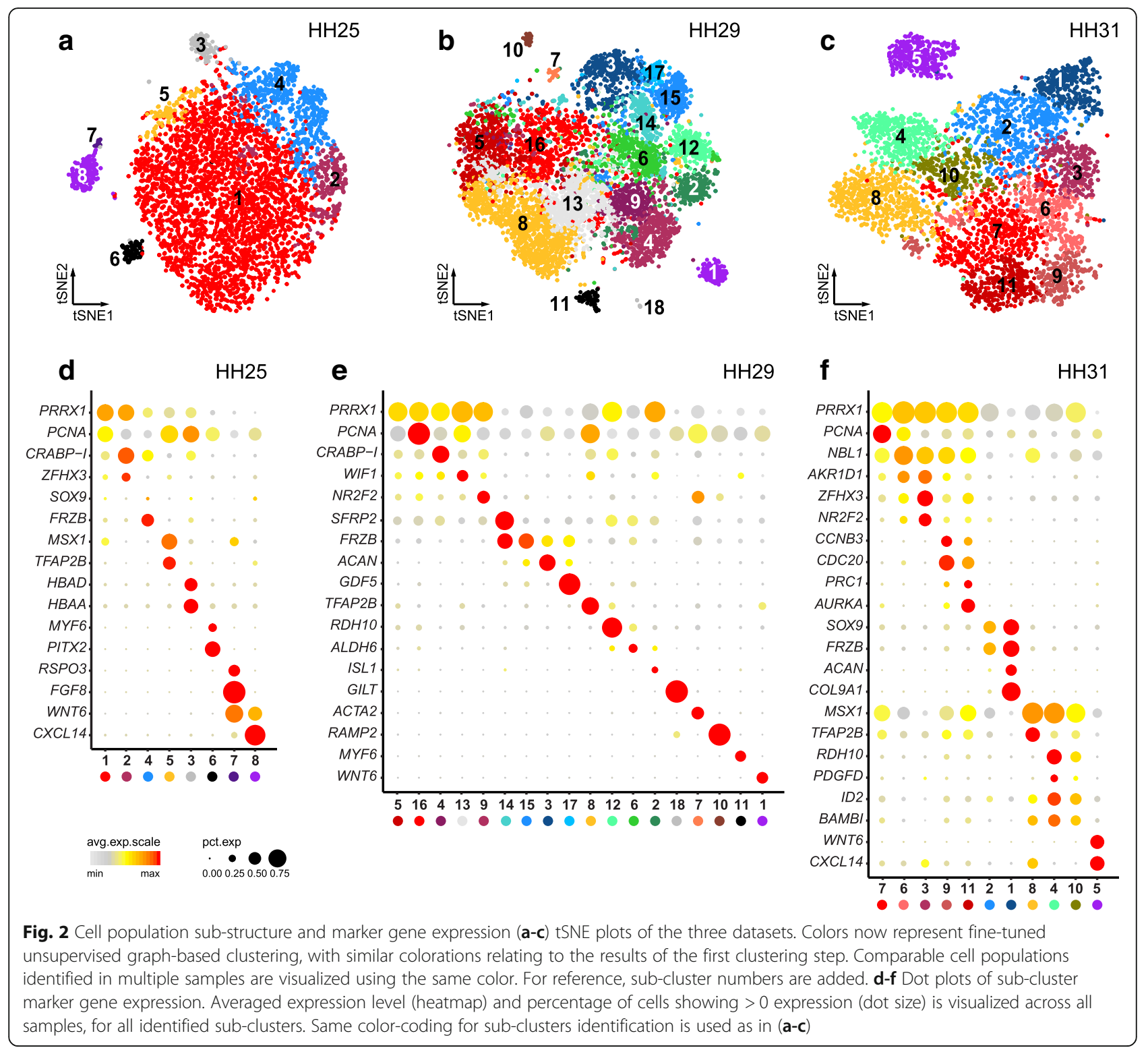

for module Green, associated with GO-terms connected to mitosis, whereas sub-cluster 16 was enriched for module Pink, linked to G2/M-transition-related genes (Additional file 1: Figure S3). Hence, we reasoned that distinct cell-cycle states underlie the subdivision of the proliferating mesenchyme cluster. Likewise, HH29 interdigit sub-clusters 2, 6 and 12 were closely matched by the activities of modules Tan, Olivegreen, Orange and Midnightblue (see below, Fig. 4a-h).

To follow the developmental dynamics of the identified modules, we calculated their averaged activities across all the three sampled time points, and visualized similarities across time and tissue types using unsupervised hierarchical clustering (Fig. 3b). Indeed, despite differences in embryonic stages and experimental platforms, we were able to confirm corresponding cell and tissue types between our samples. For example, what we refer to as the "distal mesenchyme" is a population of cells characterized by high activity of the co-expression module Magenta at all time points (Fig. 3c-f). Comparisons to published expression patterns for TFAP2B, WNT5A, MSX1 and MSX2 confirmed its distal location and, based on those genes' functions, suggested a role for this cell population in controlling distal autopod outgrowth. Using WGCNA thus enabled us to define equivalent cell populations across developmental time, and helped attribute biological functions at the sub-cluster level.

Transcriptionally and spatially distinct sub-populations in the interdigit mesenchyme

As expected by developmental stage, interdigit populations were only recovered in samples HH29 and HH31. 


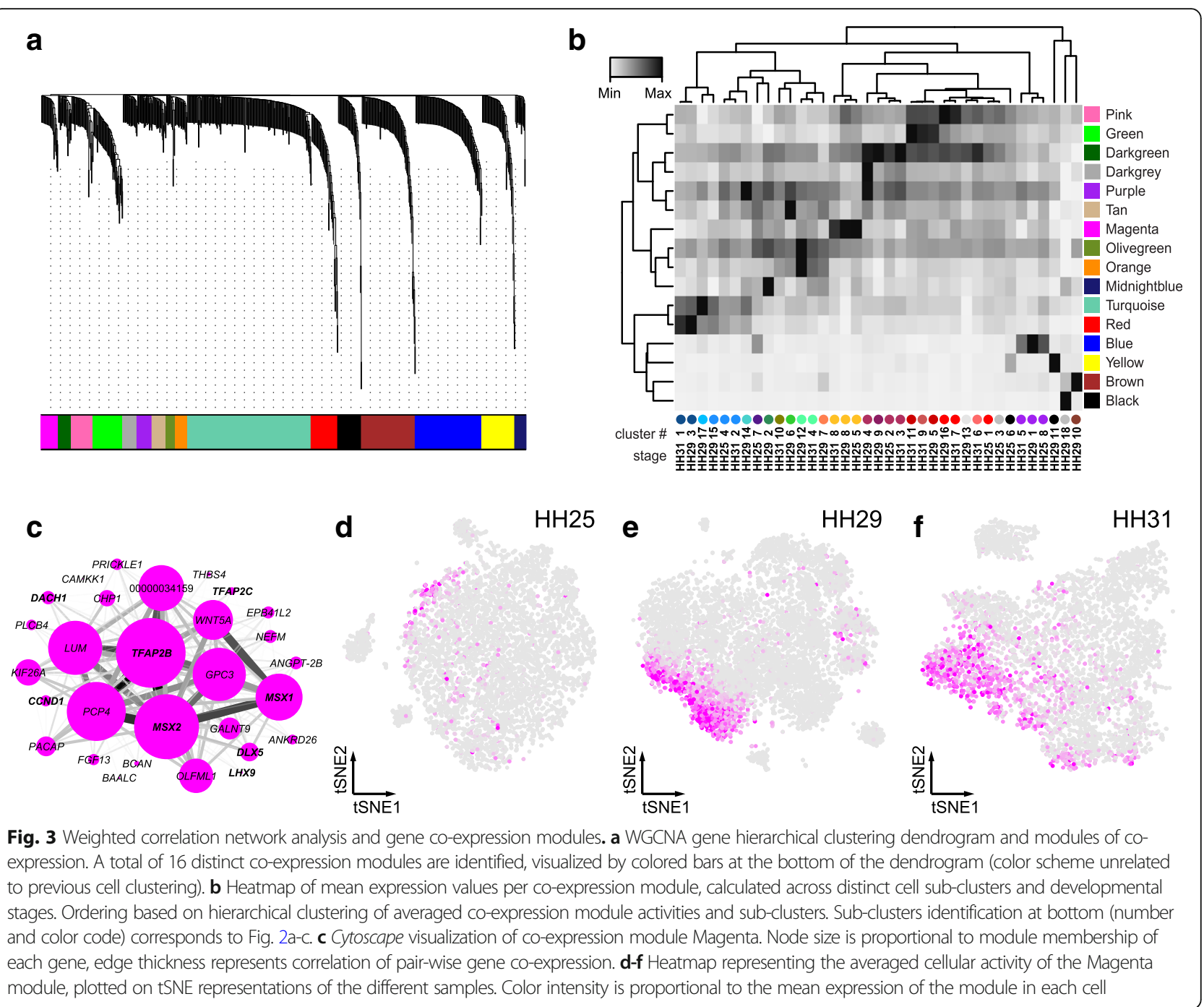

In total, we identified four associated co-expression modules (Fig. 4a-d). High Orange and Olivegreen module activities were coinciding with the same interdigit sub-population (Fig. 4e, f), which was recognizable in both HH29 and HH31 samples and marked by RDH1O expression (Fig. 2e,f). Noticeably, all genes with high membership in module Olivegreen were transcription factors (TFs), while module Orange was enriched for enzymatic activities (Fig. 4a,b). Both, however, scored high for GO-terms related to retinoic acid signaling, an important mediator of interdigit cell death [33]. Module Tan was enriched for skeletogenic and morphogenetic GO-terms, suggesting it might mediate some of the patterning information contained in the interdigit mesenchyme to the adjacently forming digits (Fig. 4c,g). Lastly, module Midnightblue showed multiple TFs and its activity was restricted to HH29 sub-cluster 2 (Fig. 4d,h).

Since relevant patterning information is contained in the interdigit, posteriorly adjacent to each forming digit, we next wondered whether some of the sub-clustering structure corresponded to spatially distinct interdigit populations along the anterior-posterior axis of the autopod. At $\mathrm{HH} 29$, we detected three interdigit sub-clusters (Fig. 4i). Using differential expression analyses, we defined marker genes that distinguish the three sub-clusters from each other (Fig. 4j). To assign putative spatial information to our single-cell interdigit transcriptomes, we reanalyzed a bulk RNA-seq dataset covering stages HH29 and HH31 of the developing chicken hindlimb autopod [34]. This dataset is based on dissections of individual digits, together with their posteriorly associated interdigit mesenchyme, and thus provided an opportunity to identify spatially resolved marker genes. We contrasted their transcriptomic data of digit/interdigit III against digit/interdigit IV and found a total of 54 genes to be significantly differentially expressed at both developmental time points (Fig. 4k). Comparing the digit/ interdigit IV-specific subset of these genes to our 


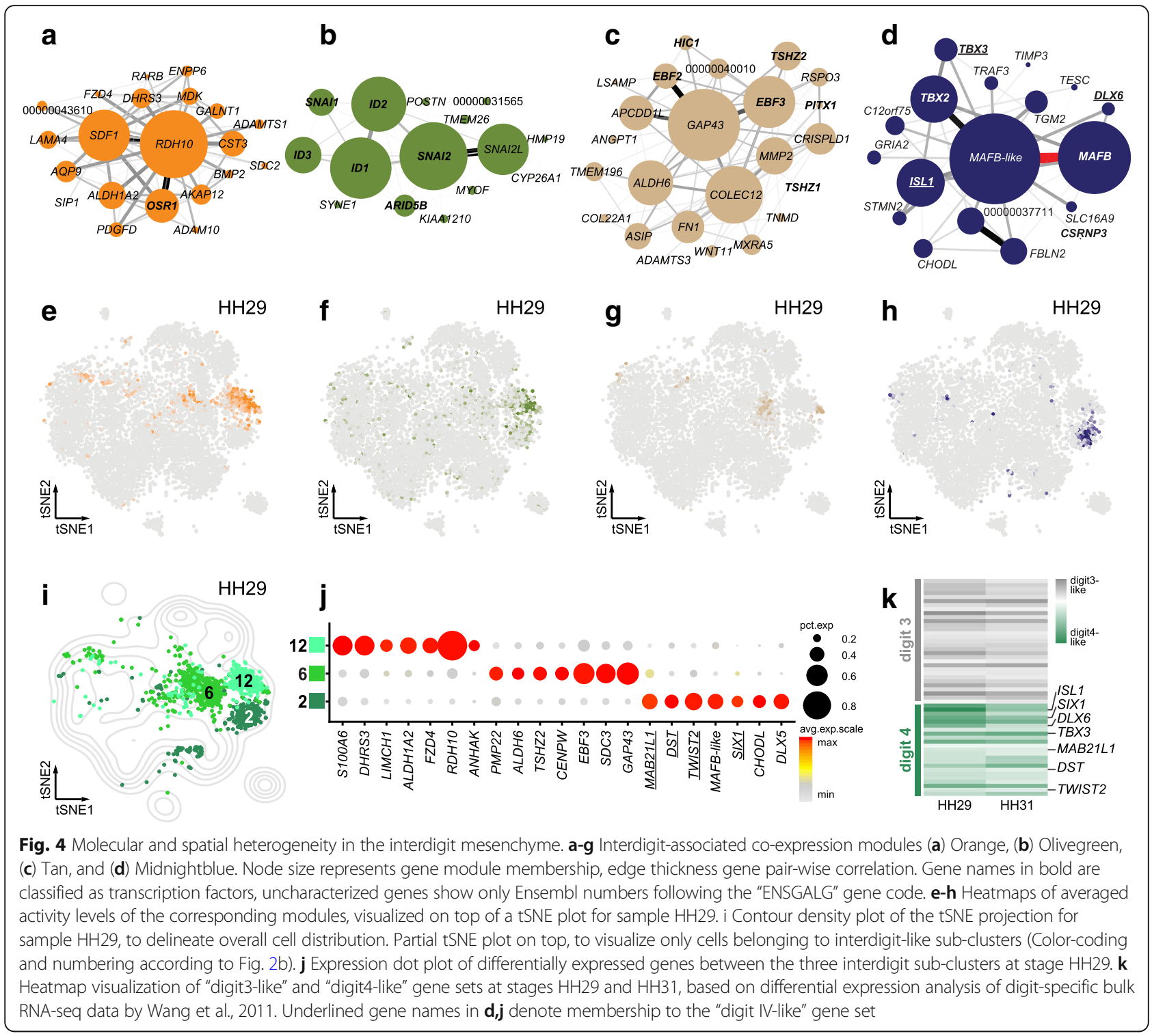

differential expression analysis of sub-cluster 2, and its affiliated module Midnightblue, we found an overlap of seven up-regulated genes (Fig. 4d,j, underlined). In contrast, we couldn't find any other digit/interdigit IV gene in the rest of the interdigit sub-cluster signatures or co-expression modules. We therefore concluded that HH29 sub-cluster 2 consisted of cells of the interdigit mesenchyme posterior to digit 4 .

\section{Developing digits and their associated tissues}

Of the cell populations directly contributing to the making of digits, a cluster reminiscent of the non-skeletal connective tissue, the nsCT, appeared in all of the samples. In our WGCNA analyses, we identified three modules, Darkgrey, Purple, and Darkgreen, which mapped to the nsCT sub-clusters (Fig. 5a-f). The Darkgrey module was most restricted, in both time and cell numbers, and its activity pattern closely matched the HH29 sub-cluster 4 (Fig. 5d). Cellular retinoic acid binding protein I CRABP-I, Aquaporin AQP1, DKK2 and GLT8D2 were the genes most strongly associated with this module. Modules Purple and Darkgreen showed more widespread activities (Fig. 5e,f), and centered on COL1A2, DCN, KCNJ2, SALL1, and AKR1D1, PRRX1, $T C F 12, Z F H X 3$. By performing stage-specific differential expression analyses for our nsCT clusters (HH25-2, HH29-9/4, HH31-3; Fig. 2), we noticed a progressive maturation of nsCT signatures, with $\mathrm{HH} 31-3$ showing the highest degree of transcriptional differentiation (Fig. 5g). Overall, however, nsCT signatures appeared developmentally dynamic and only six genes were significantly enriched across all stages (Fig. 5g), five of which also 


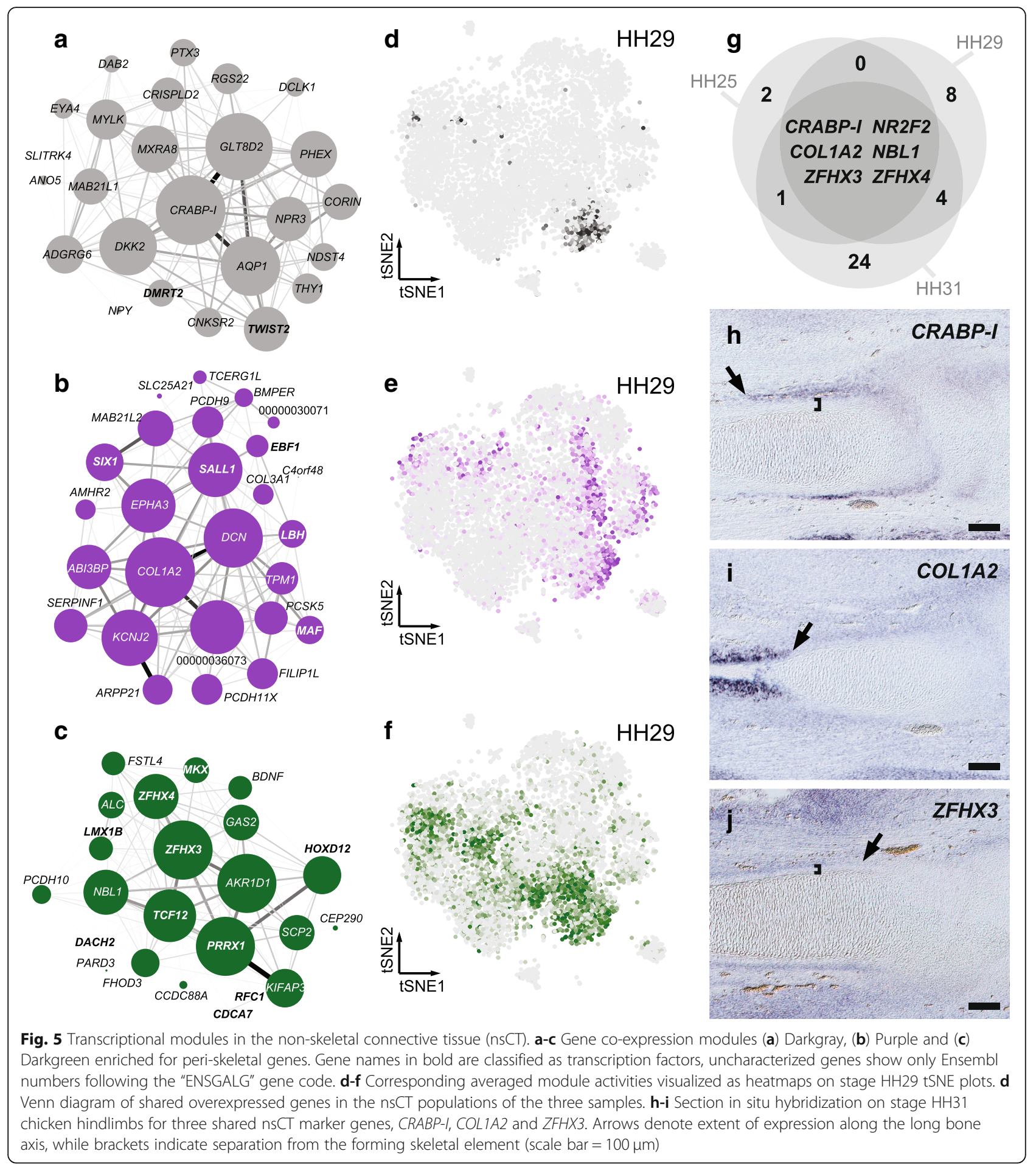

appeared in our nsCT modules. Using in situ hybridization for the top-three of these genes, both differential expressionand module membership-wise, allowed us to attribute module activities to discrete nsCT domains along the developing skeletal elements. CRABP-I showed highest expression near and around the forming epiphysis, where synovial joints and ligament attachment sites develop (Fig. 5h). COL1A2- and
ZFHX3-positive populations showed a graded distribution along the periskeletal tissue layer, predominantly marking the prospective periosteum and perichondrium domains, respectively (Fig. 5i,j).

Finally, we identified skeletal progenitor populations at all three time points (Fig. 6a-c). According to the developmental stages we sampled, only cartilage-producing 


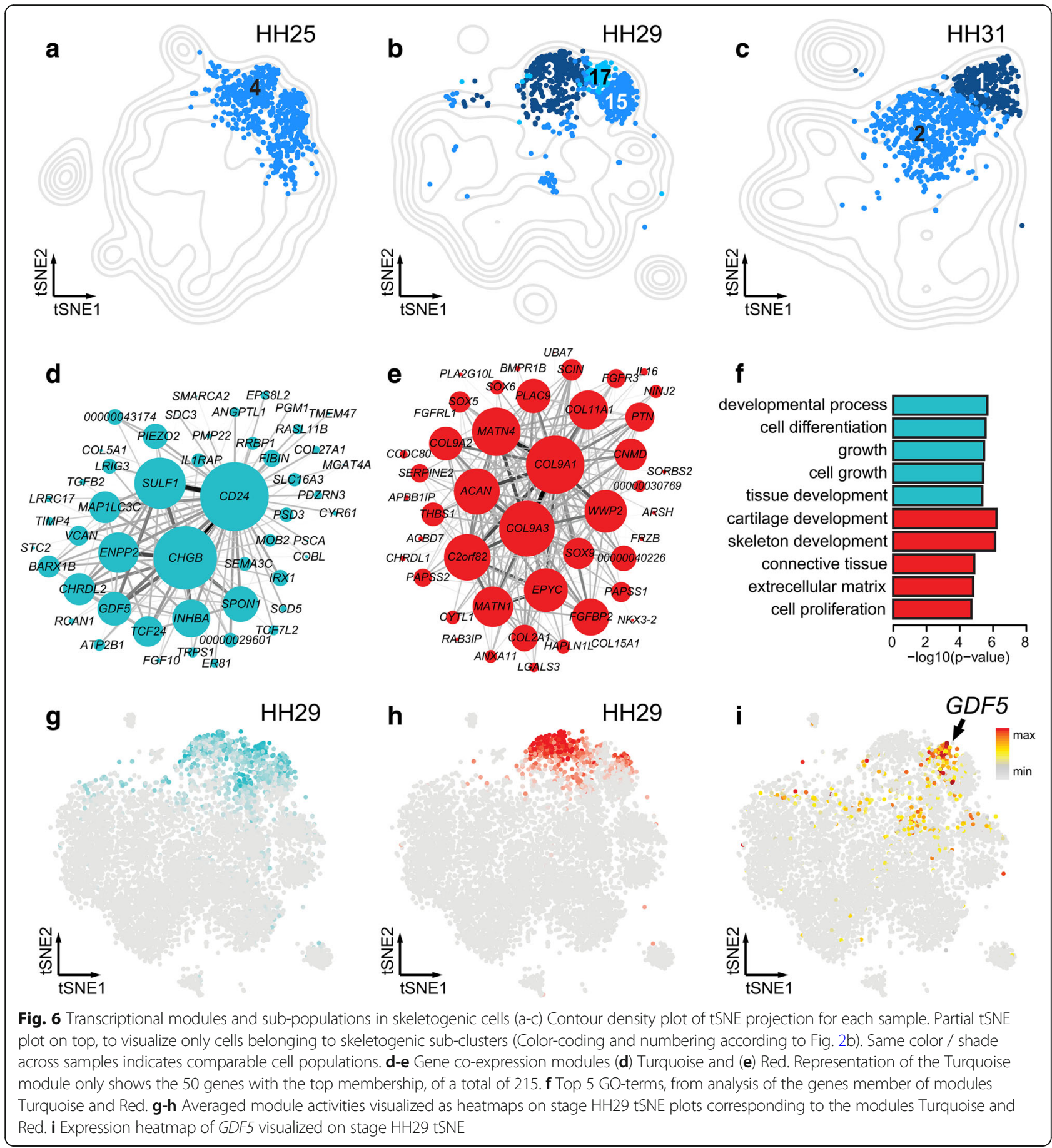

skeletal cells were recovered. In all three samples, we found a cell population resembling early chondrocytes (sub-clusters HH25-4, HH29-15 and HH31-2). At stages HH29 and HH31, a seemingly more mature chondrocyte type emerged (HH29-3, HH31-1), and an additional cartilaginous cluster was evident in the $\mathrm{HH} 29$ sample (HH29-17). Concomitantly, we identified two co-expression modules associated with these cell populations, Turquoise and Red (Fig. 6d,e). Turquoise is centered on CD24, CHGB and SULF1, whereas module Red displays a core of collagens COL9A1 and COL9A3, MATN4, C9H2ORF82 (also known as SNORC in mammals), and $A C A N$. Based on additional marker genes and GO-term enrichment analyses, we inferred the Turquoise module to be related to early chondrocyte proliferation and growth, whereas the Red module reflected chondrocyte maturation and extracellular matrix deposition (Fig. 6f). Interestingly, compared to module Turquoise, the activity of 
module Red was generally more restricted and specifically excluded from sub-cluster HH29-17 (Fig. 6g,h). Upon closer inspection, we identified high expression of several known synovial joint markers genes in this population, thus identifying it as the forming interphalangeal joints (Fig. 6i, Additional file 3).

Hence, through a combination of differential gene expression and GO-term enrichment analyses, as well as gene co-expression modules, we identified spatially and/or temporally distinct sub-populations and transcriptome dynamics in the skeletal and peri-skeletal tissues of the forming digits.

\section{Discussion}

\section{Singe-cell tissue decomposition of the developing chicken autopod}

Here, using single-cell RNA-sequencing, we present a transcriptomic atlas of the developing chicken limb at cellular resolution. Focusing on the distal and morphologically diverse portion of the limb, the autopod, we sampled over 17,000 single-cell transcriptomes with an average of over 1000 genes detected in each cell. Within our atlas, we identify all major tissue types that constitute and pattern the embryonic appendage across three developmental time points. Additionally, taking advantage of our cellular and transcriptomic sampling depth, we manage to isolate even minute cell populations like the AER and assemble lists of marker genes for them. We also distinguish transcriptionally discrete sub-populations within known major tissue types, reflecting distinct spatial locations or cellular states. As such, it demonstrates the power of scRNA-seq to molecularly disentangle cell populations of the developing limb that occur in close spatial or 'lineage' proximity. Historically, such populations have proven notoriously difficult to separate and characterize transcriptionally, using either manual tissue dissection or reporter-gene based cell lineage isolation. To what extent all of our tissue sub-clusters indeed correspond to distinct lineage separations [35], or rather represent the extremes of a molecular continuum that follows the inherently stochastic nature of transcription [36, 37], remains to be addressed in future studies. Regardless, however, our results provide a toolbox of candidate genes to tackle this question in a molecularly comprehensive manner. Furthermore, our data enables a characterization of emerging embryonic cell types based on transcriptional signatures, rather than relying on the definitive morphological and/or functional features of their mature counterparts.

\section{Cell type equivalencies across developmental and evolutionary time}

Such molecular classification schemes echo recent conceptual frameworks that aim to categorize 'cell types' across developmental and evolutionary time scales, irrespective of morphology or function [2]. If, however, we consider a 'cell type' to be primarily defined by the expression of distinct regulatory programs, then detection of program activities can substantially precede our ability to distinguish morphological or functional specializations. Indeed, our sub-clustering and module analyses across developmental time reveal the appearance of certain prospective cell types long before they become morphologically distinct. For example, already at stage HH25 we recover clear gene expression signatures reminiscent of the future periskeletal $\mathrm{nsCT}$, even though prominent cartilage anlagen have yet to form (Fig. 2d, Fig. 3b). As such, it suggests an early lineage priming, without necessarily implying a definite switch in cell fate or clear morphological distinctions. In agreement with this, our ZFHX3-containing module Darkgreen appears to be the most basic and least specific of the co-expression modules that coincide with the $\mathrm{nsCT}$ population. We detect its activity at all three time points, marking the prospective nsCT as well as parts of the PRRX1-positive mesenchymal progenitor population (Fig. 5c,f). Only later do more mature and restricted nsCT sub-divisions and their corresponding co-expression modules occur, as exemplified by the activity of module Darkgrey and some of its members known to be involved in the formation of periskeletal tissues and tendon attachment sites (Fig. 5a,d) [38, 39].

Moreover, combining such transcriptome-based 'cell type' classification schemes with comparative scRNA-seq datasets allows for a molecular assessment of homologous cell types between species, across evolutionary time scales [40, 41]. This has important implications when trying to elucidate the impact of cell type-specifying gene regulatory networks on pattern formation and diversification at its relevant cellular scale. Namely, how progenitor populations exactly perceive and process patterning-relevant cues can be modulated by species-specific alterations in the respective cell type-specifying networks. In this context, it is worth noting that we detect $R S P O 3$ as one of the main markers of the chicken AER (Fig. 2d, Additional file 2). R-spondins, a family of secreted ligands involved in WNT-signaling, have previously been implicated in AER maintenance and control of limb outgrowth. However, in mammals only RSPO2, and not RSPO3, seems to be implicated in AER function [42-44]. Similarly, species-specific modifications in the gene regulatory networks driving skeletal cell type maturation have been reported $[45,46]$. Together with recent scRNA-seq studies in other vertebrate model organisms [30,47,48], our dataset now opens new avenues for a comprehensive assessment of molecular similarities and divergences in patterning-relevant cell populations of the developing limb, across all major tetrapod clades.

\section{Digit growth and patterning at cellular resolution}

Variations in digit number, size and individual digit patterns in the autopod skeletal structure reflect functional 
specialization of tetrapod hands and feet. During development, condensations of mesenchymal cells first give rise to early skeletogenic progenitors, to then differentiate into distinct skeletal lineages such as chondrocytes, osteocytes or synovial joint cells [49-51]. However, unlike for skeletal elements at more proximal locations of the limb, individual phalanx condensations are sequentially added and expanded at the distal tip of each forming digit, through proliferation of an evolutionary conserved progenitor population [22, 23, 52]. Hence, identifying regulators of growth rates, as well as for the relative temporal sequence at which the different skeletal cell types are specified, becomes paramount when trying to understand digit-specific phalanx patterns $[25,53]$.

Early autopod outgrowth, and later digit elongation, is controlled through complex signaling interactions at the distal margin of the limb, involving the concerted action of FGFs, BMPs and WNTs (reviewed [5]). Coinciding with this distal domain, we identify a distinct sub-population of mesenchymal cell types in all of our samples, marked by elevated activity of module Magenta with TFAP2B, WNT5A and high BMP signaling (Fig. 3c-f). Certain module members have been functionally implied in regulating autopod growth and digit elongation [24, 54-56], yet others remain completely unexplored in this context.

Moreover, we identify distinct sub-populations of interdigit mesenchyme cells in our HH29 and HH31 samples, with four associated gene co-expression modules (Fig. 4a-h). Module Olivegreen contains SNAI and ID genes, known to be expressed in interdigits, and likely relates to the various BMP-driven processes in this tissue [57-62]. On the other hand, module Orange is dominated by $R D H 10$, implicated in mouse interdigital apoptosis [63]. Before its apoptotic disappearance at later stages of development, interdigit mesenchyme is known to instruct the specific phalanx-formulas of its anteriorly adjacent digit [24, 25]. Moreover, we manage to spatially attribute a distinct co-expression module (Midnightblue) to interdigit 4, i.e. posterior to a digit with known regulatory individualization in tetrapods [64].

Finally, across all developmental time points we sampled, we identify skeletogenic cell populations. At those stages, the forming skeletal elements still consist exclusively of early progenitors, maturing chondrocytes, and developing synovial joints. Accordingly, we only find three distinct sub-populations, associated with two co-expression modules. Module Red shows enrichment for many canonical markers of chondrocyte maturation (Fig. 6e) $[45,51]$. On the other hand, genes in module Turquoise do not, for the most part, evoke a classical chondrogenic transcriptional profile (Fig. 6d). Again, this module might rather reflect an early transcriptional priming, only this time towards the skeletogenic lineage. In agreement with this, we only detect low expression levels for the canonical early skeletogenic marker $S O X 9$ in $\mathrm{HH} 25$ sub-cluster 4 (Fig. 2d), which itself is specifically enriched for Turquoise activity. Likewise, our synovial joint-like HH29 sub-cluster 17 shows high activity for Turquoise, while excluding the more mature chondrocyte module Red (Fig. 6g-i).

\section{Conclusion}

Our single-cell transcriptomic atlas provides a comprehensive genomics resource to study chicken limb development in unprecedented detail. Thereby, it complements a classical experimental model of vertebrate pattern formation with molecular data at cellular resolution. We curate molecular catalogues to provide an in-depth description of the embryonic autopod, through the assembly of cell population-specific lists of candidate marker genes. Combined with the power of viral overexpression screens and recent CRISPR/Cas 9 genome modifications technologies, this resource will provide a roadmap for the functional elucidation of cell type specification programs in patterning-relevant populations. Moreover, by constructing cell population-specific gene co-expression modules, we provide a tool to follow tissue dynamics across developmental and evolutionary time scales. Thereby, it will enable insights into the molecular underpinnings of homologous cell types across all major tetrapod clades, and their ensuing developmental impact on pattern formation and diversification in the vertebrate autopod.

\section{Methods}

\section{Tissue sampling}

We collected tissue samples from embryonic hind limbs at different developmental stages (Fig. 1,a-c). Limbs were dissected in cold PBS, and chopped coarsely with a razorblade. Dissociation into single cells was done using $0.25 \%$ trypsin in DMEM and incubation for $15 \mathrm{~min}$ at $37^{\circ}$. Occasional mechanical shearing by careful pipetting was applied during the incubation time.

\section{scRNA-seq library preparation}

Single-cell suspensions of samples HH25 and HH31 were fed into a 10X Genomics Chromium Single Cell System (10X Genomics, Pleasanton, CA, USA) aiming for a concentration of 4000 cells per microliter. Cell capture, cDNA generation, preamplification and library preparation were done using Chromium Single Cell 3' $v 2$ Reagent Kit according to the manufacturer instructions. For stage $\mathrm{HH} 29$ the cells were processed with the DropSeq method according to the original protocol [26]. Once the cDNA was obtained from all the samples, the sequencing proceeded on Illumina NextSeq 500 or HiSeq 2000 platforms as recommended by the developers to an average depth of 400 million reads per sample. 


\section{Data processing}

Using either the Cell Ranger software v2 (10X Genomics) or the DropSeq pipeline v1 (https://github.com/broadinstitute/Drop-seq/releases) we performed base calling, adaptor trimming, mapping to the chicken ENSEMBL genome assembly and annotation Gallus_gallus-5.0 [65], de-multiplexing of the sequences and generation of the gene / cell count matrices.

Filtering thresholds for mapped data were adapted for each sample, depending on the different library complexities. Cells with an UMI count of more than 4 times the sample mean or less than $20 \%$ of the sample median were filtered out, cells with a mitochondrial or ribosomal contribution to UMI count of more than $10 \%$ were also filtered out. Using the R package Seurat v2.3.2 [66] the UMI counts were then Log-normalized and any variation due to the library size or mitochondrial UMI counts percentage was then regressed via a variance correction using the function ScaleData.

The cell cycle stage of each cell was inferred using the $\mathrm{R}$ package SCRAN [67] and gene pairs that covariate with cell cycle stages in mouse [68]. The gene pairs were translated to orthologous chicken genes [69] and a cell cycle stage score was obtained cell-wise for stages S, G1 and $G 2 / M$, the difference between the $G 2 / M$ and $S$ scores $(\delta G 2 M / S)$ was calculated to be accounted for in later steps.

\section{Dimensionality reduction and visualization}

Significant principal components were determined for each sample as those falling outside of a Marchenko-Pastur distribution [35]. A dimensionality reduction step was carried out, using the t-SNE algorithm [28] to visualize the data and clustering of the cells based on transcriptomic similarities. The cells were clustered using the Louvain method for community detection from large networks and the Jaccard similarity coefficient to compare similarity and diversity of the sets, implemented in the FindClusters function in Seurat using data which was additionally variance-corrected for $\delta \mathrm{G} 2 \mathrm{M} / \mathrm{S}$. A first, broad clustering step was done using a resolution of 0.4 for samples $\mathrm{HH} 31$ and $\mathrm{HH} 29$ and 0.5 for $\mathrm{HH} 25$; a second clustering was done to find sub-clusters within the data, this time using resolutions of 1.4 and 1.1 for the corresponding samples. All clustering steps were done using a $\mathrm{k}$ number of 20 and the significant principal components of the sample.

\section{Differential expression analysis}

Differential expression analyses based on the negative binomial distribution were performed with Seurat, using the $\delta G 2 \mathrm{M} / \mathrm{S}$ as a covariate and only genes expressed in at least 15\% of any compared population (Additional files 2, Additional files 3, Additional files 4); genes expressed in at least $25 \%$ of the cells and showing differences with a log fold-change $>0.5$ and an adjusted $p$ value $<0.05$ were used for GO analyses. To find expression signatures for every cell cluster, in a first step, a phylogenetic tree was obtained for the cell clusters in each sample; all directly paired clusters were tested for differential expression. Any pair of clusters with less than 15 differentially expressed genes were collapsed recursively. In a second step, specific genes for each cluster were obtained contrasting each cluster against the rest of the cells in their sample. To find genes differentially expressed genes between the interdigit clusters (Fig. 4j), we compared each of the sub-clusters against the rest of the cells in the other two clusters.

Marker genes for digit/interdigit 3 and 4 were defined using the DESeq2 R package v1.20.0 [70]. We analyzed bulk RNA data sets of digit/interdigt 3 and 4 from stage $\mathrm{HH} 28 / 29$ and HH31 of a previous study [34]. After normalization based on size factors and dispersion, we performed the differential expression analysis using a Wald test and the contrast design $\sim$ Stage+Digit to use the different stages as pseudo-replicates of the digit. We filtered for differential expression with an adjusted $p$-value $<0.05$. For visualization, we subtracted the fold changes of early and late stages and plotted a heatmap using heatmap3 R package v1.1.1 [71] using hierarchical clustering of the genes.

\section{Weighted co-expression analyses}

A weighted correlation network analysis was done using the WGCNA R package v1.6.6 [32]. Using the function FindVariableGenes from Seurat, we calculated the genes with high variation (dispersion $>0.5$ ) across all the cells in sample $\mathrm{HH} 29$, and were subsequently used in WGCNA. Adjacencies and signed topological overlaps were calculated with an inferred soft-thresholding power of 8. A hierarchical tree was constructed using the "average" method and then cut using the "tree" method at height 0.9957 and minimum module size of 15 . The eigengenes of the resulting modules, as well as the membership and a Correlation Student p value of the membership of each gene to its module were calculated. All genes not significantly ( $\mathrm{p}$ value $>0.01$ ) correlated with any module were discarded. The process was repeated recursively, until all genes were significantly associated with a module; the only change made in every iteration was the module minimum size, set to the smallest that would yield at least the same number of modules as the first analysis.

The output of WGCNA was exported to the Cytoscape v3.7.0 software [72] where the node size was coded to represent the membership, and the edge thickness and color intensity to represent the weights of each gene-pair coexpression. For visualization purposes, the scales of thickness, color and size were made relative to the minima and maxima found in each network. Furthermore, a transparency 
gradient was added to the edges, which was scaled to hide unimportant edges and avoid edge saturation, the threshold was always adjusted to make visible at least one edge per node. In only one case (module midnightblue), an edge with an outlier weight was coded to be red and thicker than any other edge, and the color/size re-scaled to the second highest weight.

\section{Gene ontology}

Gene Ontology analyses were conducted with the $R$ package limma [73]. We used the list of genes in the expression signature of each computed cell cluster, and the genes members of each co-expression module as input. For each case we used all the genes detected in the corresponding sample as the contrast universe.

\section{In situ hybridization}

Probes for CRABP-I and COL1A2 were described previously [38]. Primers for the $Z F H X 3$ probe were designed using primer3 [74]. An AA overhang and an EcoRI restriction site were added to each of the primers at the $5^{\prime}$ end. ZFHX3 (fw: [5'-AAGAATTCAGCCGTACCGGGTGCAATGAGC-3'], rev: [5'-AAGAATTCAGCGCTTCCTCTTCCCGTAGAGC-3']). In situ hybridization was performed using standard protocols [75].

\section{Additional files}

Additional file 1: Figure S1. Sample compositions and data statistics. (a) Cellular composition of the samples and datasets, color code corresponds to Fig. 2a-c. (b) UMI count distributions across the samples. (c) Gene count distributions across the samples. Figure S2. Expression patterns of marker genes. Related to Fig. 1. Normalized expression patterns of selected genes to identify the different cell populations in our broad clustering, plotted on the tSNEs from sample (a) HH25, (b) HH29 and (c) HH31. Figure S3. Co-expression modules expression patterns. Related to Fig. 3. Average expression of each WGCNA co-expression module on the tSNE of sample HH29. (PDF $4613 \mathrm{~kb}$ )

Additional file 2: Genes with enriched expression per cell population in sample HH25. Genes enriched in the different cell clusters, calculated to be differentially expressed between each cell cluster and the rest of the cells in the sample. P_val: originally calculated $p$ value; avg_logFC: average log fold-change relative to the rest of the cells; pct.x: percentage of cells in the focus cluster expressing the gene; pct.rest: percentage of cells in the rest of the clusters expressing the gene; p_val_ad: $p$ value adjusted for multiple testing; cluster: cluster number in the main text and figures; gene: ENSEMBL gene identifier; name: gene symbol, or name when available; enrichment: ratio of pct.x: pct.rest. (XLSX 153 kb)

Additional file 3: Genes with enriched expression per cell population in sample HH29. Genes enriched in the different cell clusters, calculated to be differentially expressed between each cell cluster and the rest of the cells in the sample. p_val: originally calculated $p$ value; avg_logFC: average log fold-change relative to the rest of the cells; pct.x: percentage of cells in the focus cluster expressing the gene; pct.rest: percentage of cells in the rest of the clusters expressing the gene; p_val_adj: p value adjusted for multiple testing; cluster: cluster number in the main text and figures; gene: ENSEMBL gene identifier; name: gene symbol, or name when available; enrichment: ratio of pct.X: pct.rest. (XLSX $551 \mathrm{~kb}$ )

Additional file 4: Genes with enriched expression per cell population in sample HH31. Genes enriched in the different cell clusters, calculated to be differentially expressed between each cell cluster and the rest of the cells in the sample. p_val: originally calculated $p$ value; avg_logFC: average log fold-change relative to the rest of the cells; pct.x: percentage of cells in the focus cluster expressing the gene; pct.rest: percentage of cells in the rest of the clusters expressing the gene; p_val_adj: p value adjusted for multiple testing; cluster: cluster number in the main text and figures; gene: ENSEMBL gene identifier; name: gene symbol, or name when available; enrichment: ratio of pct.x: pct.rest. (XLSX $395 \mathrm{~kb}$ )

Additional file 5: Co-expression modules and their genes. Genes part of the different co-expression modules. nodeName: ENSMBL identifier of the genes part of the module; altName: gene symbol, or name when available; membership: membership to the module. (XLSX $51 \mathrm{~kb}$ )

\section{Abbreviations}

AER: Apical ectodermal ridge; EvoDevo: Evolutionary developmental biology; GO: Gene ontology; HH: Hamburger-Hamilton stages; LPM: Lateral plate mesoderm; nsCT: Non-skeletal connective tissue; scRNA-seq: Single-cell RNA sequencing; TFs: Transcription factors; tSNE: t-distributed stochastic neighbor embedding; UMIs: Unique molecular identifiers

\section{Acknowledgements}

Calculations were performed at sciCORE (http://scicore.unibas.ch/) scientific computing center at University of Basel. CF and PT wish to acknowledge Katja Eschbach and Christian Beisel for help with 10X Genomics Chromium and sequencing. OP and PT thank Tyler Burks for help with DropSea experiments. PT and OP would like to acknowledge the generous support of Cliff Tabin and Aviv Regev, in whose labs this project was initiated (with help of $\mathrm{NIH}$ grant HD03443 to Cliff Tabin).

\section{Funding}

Work in the Tschopp laboratory is supported by the Swiss National Science Foundation (SNSF project grant 31003A_170022), the University of Basel and the Forschungsfonds of the University of Basel. These funding bodies had no role in the design of the study, collection, analysis, and interpretation of data, and in writing the manuscript.

\section{Availability of data and materials}

All data generated or analyzed during this study are included in this published article and its supplementary information files. Raw sequencing data and UMI count tables have been deposited at GEO (https://www.ncbi. nlm.nih.gov/geo/) under accession number GSE130439.

\section{Authors' contributions}

PT conceived and designed the study. CF, OP and PT conducted the scRNAseq experiments. CF conducted data analyses and in situ experiments. CF and FS conducted the bulk RNA-seq re-analysis. CF and PT drafted the manuscript. All of the authors read and approved the final manuscript.

\section{Ethics approval and consent to participate}

In accordance with Swiss national guidelines (Swiss Animal Protection Ordinance; TSchV, chapter 6, Art. 112), no formal ethics approval was required, as all experiments were carried out prior to the third trimester of incubation.

\section{Consent for publication}

Not applicable.

\section{Competing interests}

The authors declare they have no competing interests.

\section{Publisher's Note}

Springer Nature remains neutral with regard to jurisdictional claims in published maps and institutional affiliations.

\section{Author details}

'DUW Zoology, University of Basel, Vesalgasse 1, CH-4051 Basel, Switzerland. ${ }^{2}$ Klarman Cell Observatory, Broad Institute of MIT and Harvard, Cambridge, MA 02142, USA. ${ }^{3}$ Present address: The Concern Foundation Laboratories at the Lautenberg Centre for Immunology and Cancer Research, IMRIC, Hebrew University Faculty of Medicine, 91120 Jerusalem, Israel. 
Received: 7 March 2019 Accepted: 14 May 2019

\section{Published online: 22 May 2019}

\section{References}

1. Stathopoulos A, Levine M. Genomic regulatory networks and animal development. Dev Cell. 2005.

2. Arendt D, Musser JM, Baker CVH, Bergman A, Cepko C, Erwin DH, Pavlicev M, Schlosser G, Widder S, Laubichler MD, Wagner GP. The origin and evolution of cell types. Nat Rev Genet. 2016;17:744-57.

3. Moris N, Pina C, Arias AM. Transition states and cell fate decisions in epigenetic landscapes. Nat Rev Genet. 2016.

4. Eldar A, Dorfman R, Weiss D, Ashe H, Shilo DZ, Barkal N. Robustness of the BMP morphogen gradient in Drosophila embryonic patterning. Nature. 2002.

5. Zeller R, Ríos JL, Zuniga A. Vertebrate limb bud development: moving towards integrative analysis of organogenesis. Nat Rev Genet. 2009;10:845-58.

6. Perrimon N, Pitsouli C, Shilo B-Z. Signaling mechanisms controlling cell fate and embryonic patterning. Cold Spring Harb Perspect Biol. 2012;4:a005975.

7. Biesecker LG. Polydactyly: how many disorders and how many genes? 2010 update. Dev Dyn. 2011.

8. Stricker S, Mundlos S. Mechanisms of digit formation: human malformation syndromes tell the story. Dev Dyn. 2011.

9. Zuniga A, Zeller R, Probst S. The molecular basis of human congenital limb malformations. Wiley Interdiscip Rev Dev Biol. 2012.

10. Petit F, Sears KE, Ahituv N. Limb development: a paradigm of gene regulation. Nat Rev Genet. 2017;18:245-58.

11. Fedak TJ, Hall BK. Perspectives on hyperphalangy: patterns and processes. J Anat. 2004.

12. Sears KE, Behringer RR, Rasweiler JJ, Niswander LA. Development of bat flight: morphologic and molecular evolution of bat wing digits. Proc Natl Acad Sci. 2006.

13. De Bakker MAG, Fowler DA, Den OK, Dondorp EM, Carmen Garrido Navas M, Horbanczuk JO, Sire JY, Szczerbińska D, Richardson MK. Digit loss in archosaur evolution and the interplay between selection and constraints. Nature. 2013.

14. Cooper KL, Sears KE, Uygur A, Maier J, Baczkowski KS, Brosnahan M, Antczak D, Skidmore JA, Tabin CJ. Patterning and post-patterning modes of evolutionary digit loss in mammals. Nature. 2014.

15. Wachtler F, Christ B, Jacob HJ. On the determination of mesodermal tissues in the avian embryonic wing bud. Anat Embryol (Berl). 1981.

16. Logan M, Martin JF, Nagy A, Lobe C, Olson EN, Tabin CJ. Expression of Cre recombinase in the developing mouse limb bud driven by a Prxl enhancer. Genesis. 2002.

17. Pearse RV, Scherz PJ, Campbell JK, Tabin CJ. A cellular lineage analysis of the chick limb bud. Dev Biol. 2007.

18. Chevallier A, Kieny M, Mauger A. Limb-somite relationship: origin of the limb musculature. J Embryol Exp Morphol. 1977.

19. Christ B, Jacob HJ, Jacob M. Experimental analysis of the origin of the wing musculature in avian embryos. Anat Embryol (Berl). 1977.

20. Riddle RD, Johnson RL, Laufer $\mathrm{E}$, Tabin $\mathrm{C}$. Sonic hedgehog mediates the polarizing activity of the ZPA. Cell. 1993.

21. Lopez-Rios J, Duchesne A, Speziale D, Andrey G, Peterson KA, Germann P, et al. Attenuated sensing of SHH by Ptch1 underlies evolution of bovine limbs. Nature. 2014.

22. Montero JA, Lorda-Diez Cl, Gañan Y, Macias D, Hurle JM. Activin/TGF $\beta$ and BMP crosstalk determines digit chondrogenesis. Dev Biol. 2008

23. Suzuki T, Hasso SM, Fallon JF. Unique SMAD1/5/8 activity at the phalanxforming region determines digit identity. Proc Natl Acad Sci U S A. 2008; 105:4185-90.

24. Dahn RD, Fallon JF. Interdigital regulation of digit identity and homeotic transformation by modulated BMP signaling. Science (80- ); 2000.

25. Huang BL, Trofka A, Furusawa A, Norrie JL, Rabinowitz AH, Vokes SA, Taketo MM, Zakany J, Mackem S. An interdigit signalling Centre instructs coordinate phalanx-joint formation governed by $5^{\prime}$ Hoxd-Gli3 antagonism. Nat Commun. 2016;7:1-10

26. Macosko EZ, Basu A, Satija R, Nemesh J, Shekhar K, Goldman M, Tirosh I, Bialas AR, Kamitaki N, Martersteck EM, Trombetta JJ, Weitz DA, Sanes JR, Shalek AK, Regev A, McCarroll SA. Highly parallel genome-wide expression profiling of individual cells using Nanoliter droplets. Cell. 2015;161:1202-14.

27. Zheng GXY, Terry JM, Belgrader P, Ryvkin P, Bent ZW, Wilson R, et al. Massively parallel digital transcriptional profiling of single cells. Nat Commun. 2017:8:1-12.
28. Van Der Maaten LJP, Hinton GE. Visualizing high-dimensional data using tsne. J Mach Learn Res. 2008;9:2579-605.

29. Cserjesi P, Lilly B, Bryson L, Wang Y, Sassoon DA, Olson EN. MHox: a mesodermally restricted homeodomain protein that binds an essential site in the muscle creatine kinase enhancer. Development. 1992.

30. Gerber T, Murawala P, Knapp D, Masselink W, Schuez M, Hermann S, GacSantel M, Nowoshilow S, Kageyama J, Khattak S, Currie JD, Camp JG, Tanaka EM, Treutlein B. Single-cell analysis uncovers convergence of cell identities during axolotl limb regeneration. Science. 2018;(80):362.

31. Bravo R, Frank R, Blundell PA, Macdonald-Bravo H. Cyclin/PCNA is the auxiliary protein of DNA polymerase- $\delta$. Nature. 1987.

32. Langfelder $P$, Horvath S. WGCNA: an R package for weighted correlation network analysis. BMC Bioinformatics. 2008;9.

33. Dupé V, Ghyselinck NB, Thomazy V, Nagy L, Davies PJA, Chambon P, Mark $M$. Essential roles of retinoic acid signaling in interdigital apoptosis and control of BMP-7 expression in mouse autopods. Dev Biol. 1999.

34. Wang Z, Young RL, Xue H, Wagner GP. Transcriptomic analysis of avian digits reveals conserved and derived digit identities in birds. Nature. 2011; 477:583-7.

35. Shekhar K, Lapan SW, Whitney IE, Tran NM, Macosko EZ, Kowalczyk M, Adiconis X, Levin JZ, Nemesh J, Goldman M, McCarroll SA, Cepko CL, Regev A, Sanes JR. Comprehensive classification of retinal bipolar neurons by single-cell transcriptomics. Cell. 2016;166:1308-1323.e30.

36. Nicolas D, Phillips NE, Naef F. What shapes eukaryotic transcriptional bursting? Mol BioSyst. 2017.

37. Urban EA, Johnston RJ. Buffering and Amplifying Transcriptional Noise During Cell Fate Specification. Front Genet. 2018;9 November:1-14.

38. Bandyopadhyay A, Kubilus JK, Crochiere ML, Linsenmayer TF, Tabin CJ. Identification of unique molecular subdomains in the perichondrium and periosteum and their role in regulating gene expression in the underlying chondrocytes. Dev Biol. 2008.

39. Witte F, Dokas J, Neuendorf F, Mundlos S, Stricker S. Comprehensive expression analysis of all Wnt genes and their major secreted antagonists during mouse limb development and cartilage differentiation. Gene Expr Patterns. 2009.

40. Marioni JC, Arendt D. How single-cell genomics is changing evolutionary and developmental biology. Annu Rev Cell Dev Biol. 2017:33:537-53.

41. Tschopp P, Tabin CJ. Deep homology in the age of next-generation sequencing. Philosophical Transactions of the Royal Society B: Biological Sciences. 2017.

42. Nam JS, Park E, Turcotte TJ, Palencia S, Zhan X, Lee J, Yun K, Funk WD, Yoon JK. Mouse R-spondin2 is required for apical ectodermal ridge maintenance in the hindlimb. Dev Biol. 2007.

43. Neufeld S, Rosin JM, Ambasta A, Hui K, Shaneman V, Crowder R, Vickerman $\mathrm{L}$, Cobb J. A conditional allele of Rspo3 reveals redundant function of Rspondins during mouse limb development. Genesis. 2012.

44. Szenker-Ravi E, Altunoglu U, Leushacke M, Bosso-Lefèvre C, Khatoo M, Thi Tran $\mathrm{H}$, et al. RSPO2 inhibition of RNF43 and ZNRF3 governs limb development independently of LGR4/5/6. Nature. 2018.

45. Gómez-Picos P, Eames BF. On the evolutionary relationship between chondrocytes and osteoblasts. Front Genet. 2015.

46. Ferguson GB, Van Handel B, Bay M, Fiziev P, Org T, Lee S, et al. Mapping molecular landmarks of human skeletal ontogeny and pluripotent stem cellderived articular chondrocytes. Nat Commun. 2018.

47. Fabre PJ, Leleu M, Mascrez B, Lo GQ, Cobb J, Duboule D. Single-cell mRNA profiling reveals heterogeneous combinatorial expression of Hoxd genes during limb development. BMC Biol. 2018:327619.

48. Cao J, Spielmann M, Qiu X, Huang X, Ibrahim DM, Hill AJ, Zhang F, Mundlos S, Christiansen L, Steemers FJ, Trapnell C, Shendure J. The single-cell transcriptional landscape of mammalian organogenesis. Nature. 2019.

49. Hartmann C, Tabin CJ. Wnt-14 plays a pivotal role in inducing synovial joint formation in the developing appendicular skeleton. Cell. 2001.

50. Akiyama H, Kim J-E, Nakashima K, Balmes G, Iwai N, Deng JM, Zhang Z, Martin JF, Behringer RR, Nakamura T, de Crombrugghe B. Osteochondroprogenitor cells are derived from Sox9 expressing precursors. Proc Natl Acad Sci. 2005.

51. Kozhemyakina E, Lassar AB, Zelzer E. A pathway to bone: signaling molecules and transcription factors involved in chondrocyte development and maturation. Development. 2015.

52. Witte F, Chan D, Economides AN, Mundlos S, Stricker S. Receptor tyrosine kinaselike orphan receptor 2 (ROR2) and Indian hedgehog regulate digit outgrowth mediated by the phalanx-forming region. Proc Natl Acad Sci. 2010. 
53. Hiscock TW, Tschopp P, Tabin CJ. On the formation of digits and joints during limb development. Dev Cell. 2017.

54. Sanz-Ezquerro JJ, Tickle C. Fgf signaling controls the number of phalanges and tip formation in developing digits. Curr Biol. 2003.

55. Gros J, Hu JKH, Vinegoni C, Feruglio PF, Weissleder R, Tabin CJ. WNT5A/JNK and FGF/MAPK pathways regulate the cellular events shaping the vertebrate limb bud. Curr Biol. 2010.

56. Seki R, Kitajima K, Matsubara H, Suzuki T, Saito D, Yokoyama H, Tamura K. $\mathrm{AP}-2 \beta$ is a transcriptional regulator for determination of digit length in tetrapods. Dev Biol. 2015

57. Jen Y, Manova K, Benezra R. Expression patterns of $\mathrm{Id} 1$, Id2, and Id 3 are highly related but distinct from that of Id4 during mouse embryogenesis. Dev Dyn. 1996.

58. Ros MA, Sefton M, Nieto MA. Slug, a zinc finger gene previously implicated in the early patterning of the mesoderm and the neural crest, is also involved in chick limb development. Development. 1997.

59. Nieto MA. The snail superfamily of zinc-finger transcription factors. Nat Rev Mol Cell Biol. 2002

60. Zuzarte-Luís V, Hurlé JM. Programmed cell death in the developing limb. Int J Dev Biol. 2002

61. Lorda-Diez Cl, Torre-Pérez N, García-Porrero JA, Hurle JM, Montero JA. Expression of Id2 in the developing limb is associated with zones of active BMP signaling and marks the regions of growth and differentiation of the developing digits. Int J Dev Biol. 2009.

62. Pignatti E, Zeller R, Zuniga A. To BMP or not to BMP during vertebrate limb bud development. Semin Cell Dev Biol. 2014.

63. Cunningham TJ, Chatzi C, Sandell LL, Trainor PA, Duester G. Rdh10 mutants deficient in limb field retinoic acid signaling exhibit normal limb patterning but display interdigital webbing. Dev Dyn. 2011.

64. Stewart TA, Liang C, Cotney J, Noonan JP, Sanger T, Wagner G. Evidence against tetrapod-wide digit identities and for a limited frame shift in bird wings. bioRxiv. 2018:224147.

65. Zerbino DR, Achuthan P, Akanni W, Amode MR, Barrell D, Bhai J, et al. Ensembl 2018. Nucleic Acids Res. 2017;2017:1-8.

66. Butler A, Hoffman P, Smibert P, Papalexi E, Satija R. Integrating single-cell transcriptomic data across different conditions, technologies, and species. Nat Biotechnol. 2018.

67. Lun ATL, McCarthy DJ, Marioni JC. A step-by-step workflow for low-level analysis of single-cell RNA-seq data with Bioconductor. F1000Research. 2016;5:2122

68. Scialdone A, Natarajan KN, Saraiva LR, Proserpio V, Teichmann SA, Stegle O, Marioni JC, Buettner F. Computational assignment of cell-cycle stage from single-cell transcriptome data. Methods. 2015;85:54-61.

69. Durinck S, Spellman PT, Birney E, Huber W. Mapping identifiers for the integration of genomic datasets with the R/ Bioconductor package biomaRt. Nat Protoc. 2009:4:1184-91.

70. Love Ml, Huber W, Anders S. Moderated estimation of fold change and dispersion for RNA-seq data with DESeq2. Genome Biol. 2014.

71. Zhao S, Guo Y, Sheng Q, Shyr Y. Heatmap3: an improved heatmap package with more powerful and convenient features. BMC Bioinformatics. 2014

72. Shannon P, Markiel A, Ozier O, Baliga NS, Wang JT, Ramage D, Amin N, Schwikowski B, Ideker T. Cytoscape: a software environment for integrated models of biomolecular interaction networks. Genome Res. 2003.

73. Ritchie ME, Phipson B, Wu D, Hu Y, Law CW, Shi W, Smyth GK. Limma powers differential expression analyses for RNA-sequencing and microarray studies. Nucleic Acids Res. 2015;43:e47.

74. Rozen S, Skaletsky H. In: Krawetz S, Misener S, editors. Methods and protocols: methods in molecular biology Primer3 on the WWW for general users and for biologist programmers. Totowa, NJ: Humana Press; 2000.

75. McGlinn E, Mansfield JH. Detection of gene expression in mouse embryos and tissue sections. In: Pelegri FJ, editor. Vertebrate embryogenesis: embryological, cellular, and genetic methods. Totowa, NJ: Humana Press; 2011. p. 259-92.

Ready to submit your research? Choose BMC and benefit from:

- fast, convenient online submission

- thorough peer review by experienced researchers in your field

- rapid publication on acceptance

- support for research data, including large and complex data types

- gold Open Access which fosters wider collaboration and increased citations

- maximum visibility for your research: over $100 \mathrm{M}$ website views per year

At BMC, research is always in progress.

Learn more biomedcentral.com/submissions 